\title{
Treatment Strategy and Outcomes in Patients with Hematogenous Culture-Negative Pyogenic Vertebral Osteomyelitis
}

\author{
Gouse Mohamad, Rohit Amritanand, Kenny Samuel David, Venkatesh Krishnan, Justin Arockiaraj \\ Spinal Disorders Surgery Unit, Department of Orthopaedics, Christian Medical College, Vellore, India
}

\begin{abstract}
Study Design: Retrospective case series.
Purpose: The aim of this study was to analyze functional and radiological outcomes in patients with culture-negative pyogenic vertebral osteomyelitis (PVO).

Overview of Literature: There were only few literature available for these group of patients.

Methods: Patients with biopsy-positive but culture-negative PVO were included. We analyzed records for data on demography, comorbidities, coexisting infections, neurological status, prior antibiotic therapy, pre- and postoperative erythrocyte sedimentation rate, C-reactive protein levels, and Oswestry Disability Index, and Japanese Orthopedics Association scores.

Results: Sixty-one patients were included, of which data of 45 patients were available for follow-up. The patients were predominantly males $(71 \%)$, with a mean age of 53.2 years. Seventy-seven percent patients had comorbidities. Echocardiography, blood culture, and urine culture were performed on $8 \%, 24 \%$, and $18 \%$ of patients, respectively. Thirty-one percent patients had neurological deficits. Computed tomography-guided biopsy was performed on $67 \%$ patients. Fifty-two percent patients were treated surgically, and $48 \%$ were treated non-surgically. Nineteen percent patients were treated according to the results of cultures from other foci, and the rest were treated empirically. For the initial 2 weeks, all patients were treated with intravenous antibiotics empirically or based on culture from other foci. This treatment was followed by 10 weeks of oral cloxacillin/cephalexin for gram-positive organisms or ciprofloxacin for gram-negative organisms. The mean follow-up time was 18 months (range, 12-120 months). All patients had improvement in Japanese Orthopedics Association, Oswestry Disability Index, and Visual Analog Scale scores $(p<0.001)$.

Conclusions: Treatment with empirical antibiotics for 12 weeks with watchful clinical and radiological follow-up yields good resolution of the disease. Further multicenter clinical research needs to be performed for obtaining an algorithmic treatment plan for these patients.
\end{abstract}

Keywords: Pyogenic spondylodiscitis; Culture negative pyogenic infection

\section{Introduction}

Management of pyogenic vertebral osteomyelitis (PVO) is a challenging problem in spite of advances in imaging, di- agnostic, and treatment modalities. The incidence of pyogenic infection in the literature is approximately 1:250,000, and there is a recent trend toward an increase in the incidence of infection [1,2]. The incidence of culture-negative

Received Apr 19, 2018; Revised May 27, 2018; Accepted Jul 1, 2018

Corresponding author: Gouse Mohamad

Spinal Disorders Surgery Unit, Department of Orthopaedics, Christian Medical College, Vellore, Tamil Nadu 632004, India

Tel: +91-7598387281, Fax:+91-0416-2232035, E-mail: gousemohamad@yahoo.com 
PVO was 0.3-1.8 cases per 100,000 [3]. Regardless of the treatment protocol, identification of the causative organism is crucial for selecting the antibiotic regime. Although surgical debridement helps in de-bulking the bacterial load, the need for antibiotic administration for preventing recurrence of the disease is paramount. Establishing a definitive diagnosis and managing the disease may be difficult when the culture specimen does not grow specific pathogens during histopathological examination suggestive of pyogenic infection. There are many reports describing the management and outcome of culture-positive PVO. However, there are few reports that describe the antibiotic protocol or functional outcome of culture-negative PVO. The aim of this study was to analyze the antibiotic protocols and functional and radiological outcomes in patients with hematogenous culture-negative PVO.

\section{Materials and Methods}

This study was approved by the institutional review board of Christian Medical College, Vellore, India (IRB Min no., 10216). Informed consent obtained from all patients. The study was conducted at a tertiary care spine referral center. We included patients who were pus culture-negative with histopathological features suggestive of PVO (i.e., spicules of necrotic bone with trabeculae of cortical and cancellous bone with marrow replaced by inflammatory granulation tissue comprising mild to moderate infiltrates of plasma cells, lymphocytes, eosinophils, and neutrophils with no granulomas). The patients were either treated conservatively or surgically from January 2005 to December 2015.

We retrospectively analyzed online medical records, patient charts, operative notes, radiographs, and discharge summaries. Patient demographic data, comorbidities, coexisting infections, level of spine involvement, pretreatment neurological status, and prior antibiotic therapy were documented. Inflammatory markers, such as erythrocyte sedimentation rate (ESR), total leukocyte count, and C-reactive protein (CRP), were documented pre- and postoperatively. Blood and urine culture, echocardiography for cardiac vegetations, and ultrasonography to look for intrapelvic abscess were performed. Our indications for surgery were progressive neurological deficit, mechanical instability, pain that did not subside with analgesics, and failed computed tomography (CT)-guided biopsy. Tissue samples were obtained by either CT-guided biopsy or open biopsy. The Visual Analog Scale (VAS) score, the Oswestry Disability Index (ODI), and the Japanese Orthopedics Association (JOA) score were documented preoperatively and on subsequent follow-up. At final follow-up, radiological imaging to assess fusion and healing status was performed, in addition to obtaining clinical scores.

Categorical variables were expressed as frequencies and percentages. Continuous variables were summarized as means and standard deviations. Pre- and postoperative ODI, JOA, and VAS scores were compared by the Wilcoxon signed rank test, as the distribution was not symmetric. A $p$-value $<0.05$ was considered to indicate statistical significance. The data were analyzed with the SPSS ver. 17.0 (SPSS Inc., Chicago, IL, USA).

\section{Results}

A total of 61 patients were included in the study, of whom 43 (71\%) were males. The mean age was $53.2 \pm 1.7$ years. Twelve patients (19\%) were lost to follow-up, and four patients $(6 \%)$ died from unassociated medical conditions after completion of treatment. Thus, complete followup was possible on 45 patients (73\%). Forty-one patients (77\%) had comorbidities. Forty-eight patients (79\%) had symptoms for less than 2 months. Thirty-one patients (51\%) did not report any previous source of infection. Prior to presentation, 20 patients (32\%) had received an

Table 1. Baseline characteristics

\begin{tabular}{lc} 
Characteristic & No. (\%) \\
Sex & \\
\hline Male & $43(70.5)$ \\
Female & $18(29.5)$ \\
Comorbidities & \\
\hline None & $20(32.8)$ \\
\hline Diabetes mellitus & $25(41.0)$ \\
\hline Liver disease & $1(1.6)$ \\
\hline Kidney disease & $4(6.6)$ \\
\hline Others & $11(18.0)$ \\
\hline Smoking & \\
\hline Yes & $9(14.8)$ \\
\hline No & $52(85.2)$ \\
\hline Alcohol & \\
\hline Yes & $3(4.9)$ \\
\hline No & $58(95.1)$ \\
\hline
\end{tabular}


initial course of antibiotics from the primary treating physician. Fifteen (24\%), 11 (18\%), and five patients (8\%) underwent blood culture, urine culture, and echocardiography, respectively. Baseline characteristics of the patients are presented in Table 1. The radiological findings and levels of spine involvement are shown in Tables 2 and 3, respectively. Nineteen patients (31\%) had neurological deficits at presentation; 12 of these 19 patients (63\%) were available for final follow-up, and all recovered from the

Table 2. Radiological findings among the patients

\begin{tabular}{lc} 
Radiological finding & No. $(\%)$ \\
X-ray features & \\
\hline Vertebral erosion & $38(62.3)$ \\
\hline Vertebral collapse & $18(29.5)$ \\
\hline Other findings & $5(8.0)$ \\
\hline Magnetic resonance imaging findings \\
\hline Epidural abscess \\
\hline Disk space collapse & $21(34.4)$ \\
\hline Others & $37(60.7)$ \\
\hline
\end{tabular}

Table 3. Levels of spine involvement

\begin{tabular}{lc} 
Level of spine involvement & No. $(\%)$ \\
Cervical & $2(3.3)$ \\
\hline Thoracolumbar & $15(24.6)$ \\
\hline Lumbar & $33(54.1)$ \\
\hline Lumbosacral & $11(18.0)$ \\
\hline
\end{tabular}

Table 4. Preoperative and postoperative neurological pattern

\begin{tabular}{lcc} 
Frankel grade & Preoperative & Postoperative \\
\hline A & 0 & 0 \\
B & 0 & 0 \\
\hline C & 3 & 0 \\
D & 12 & 0 \\
\hline E & 30 & 45 \\
\hline Total & 45 & 45 \\
\hline
\end{tabular}

deficit (Table 4). Forty-one patients (67\%) had CT-guided biopsy. Thirty-one patients (51\%) had surgical treatment with instrumented fusion, and 30 patients (49\%) had conservative treatment based on the report from CT-guided biopsy. Forty-two patients (70\%) were started empirically on antibiotics. Twelve (19\%) and seven patients (11\%) were started on antibiotics on the basis of culture from other remote foci or discussion with an infectious disease specialist, respectively.

The mean duration of follow-up was 18 months, with a range of $12-120$ months. Forty-five patients (73\%) were available for the final follow-up. The mean preoperative ODI score was $44.2 \pm 1.5$; the mean postoperative score dropped significantly to $11.9 \pm 33(p<0.001)$. Similarly, the JOA and VAS scores showed significant improvement between the pre- and postoperative period ( $p<0.001$ for both comparisons) (Table 5). The mean preoperative ESR and CRP levels were $55 \mathrm{~mm} / \mathrm{hr}$ (range, 40 to $82.5 \mathrm{~mm} /$ $\mathrm{hr}$ ) and $40 \mathrm{mg} / \mathrm{L}$ (range, 25 to $60.5 \mathrm{mg} / \mathrm{L}$ ), respectively. The ESR and CRP levels also dropped significantly in the postoperative period to $17 \mathrm{~mm} / \mathrm{hr}$ (range, 10 to $25 \mathrm{~mm} /$ $\mathrm{hr}$ ) and $3 \mathrm{mg} / \mathrm{L}$ (range, 3 to $5 \mathrm{mg} / \mathrm{L}$ ), respectively. All patients had clinical and radiological bony fusion at the final follow-up, as assessed by two independent surgeons. Case illustrations of one patient were described in Figs. 1-6. One patient had persistent sinus at the lower back. One patient had screw cut-out into the superior disk space, although the infected segment united well. Implant removal has been planned for this patient. One patient had persistent pain due to mechanical instability after completion of 3 months of empirical antibiotics. After evaluation, he underwent posterior instrument fusion, followed by the same empirical antibiotics, which went on to union.

\section{Discussion}

This series describes the antibiotic protocol and the functional and radiological outcomes of culture-negative, biopsy-positive PVO. The current cohort of patients showed

Table 5. Preoperative and postoperative functional outcome measure

\begin{tabular}{lccc} 
Variable & Preoperative & Postoperative & $p$-value \\
Visual Analog Scale score & $7.7 \pm 0.8$ & $3.3 \pm 0.6$ & $<0.001$ \\
Oswestry Disability Index score & $44.2 \pm 1.5$ & $11.8 \pm 3.4$ & $<0.001$ \\
Japanese Orthopedics Association score & $7.8 \pm 2.3$ & $25.6 \pm 1.9$ & $<0.001$ \\
\hline
\end{tabular}

Values are presented as mean \pm standard deviation. 


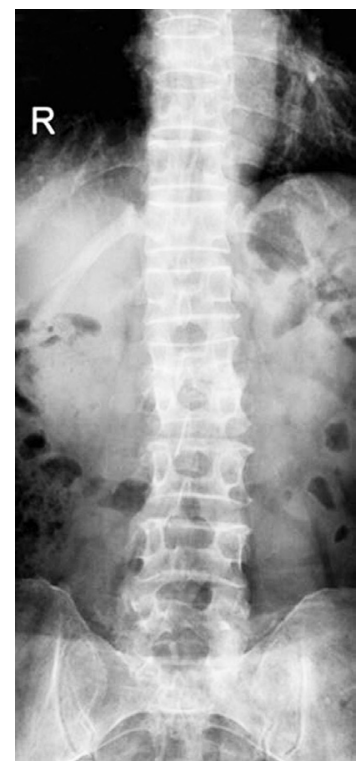

Fig. 1. Preoperative anteroposterior view with decreased height of the vertebral body at the L1-L2 level.

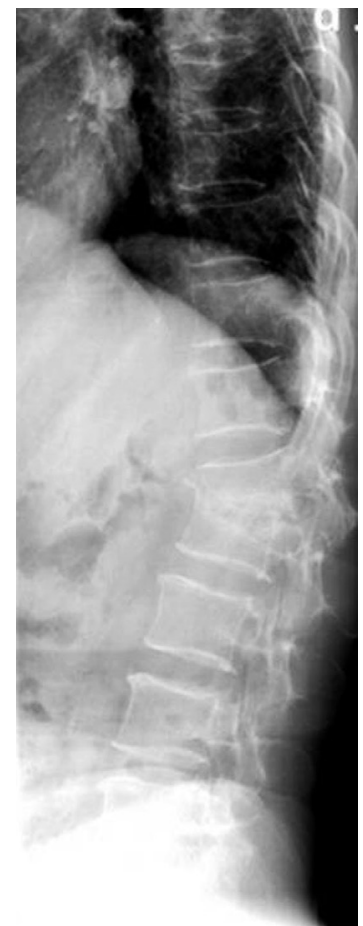

Fig. 2. Preoperative lateral view of $\mathrm{L} 1-\mathrm{L} 2$ infective spondylodiskitis with erosion of the intervertebral disk and body.

significant improvements in VAS, functional outcomes such as JOA and ODI, and good radiological union. All patients were empirically treated with first-generation antibiotics. The most commonly involved spine level was the lumbar (54\%) followed by the thoracic (25\%), lumbosacral (18\%), and cervical (4\%) levels, a finding similar

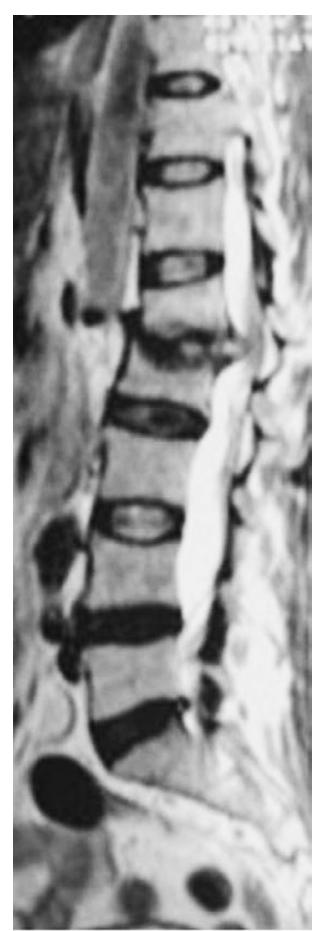

Fig. 3. T2W image showing hyperintensity at the disk with epidural compression of the dura.

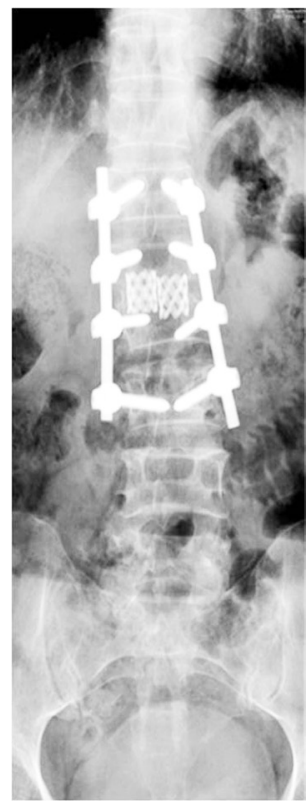

Fig. 4. Follow-up anteroposterior radiographs with solid fusion with implant in situ.

to those in other published studies of PVO [4]. Fiftyone percent of patients underwent surgical debridement and posterior instrumented fusion, and $49 \%$ were treated conservatively with antibiotics alone. Thirty-two percent of patients had neurological deficits preoperatively, and 


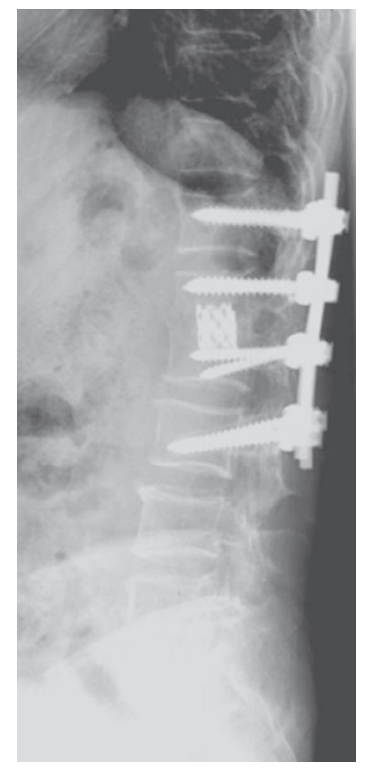

Fig. 5. Follow-up lateral radiographs with solid fusion with implant in situ.

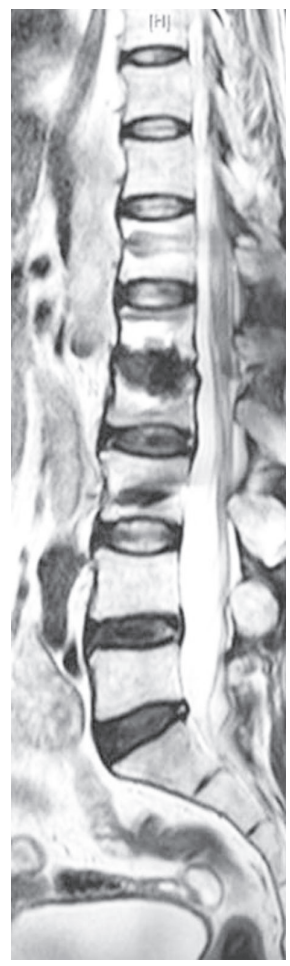

Fig. 6. T2W sagittal cut shows complete resolution of the disease.

the functional neurology of all patients was improved at the final follow-up. Five percent of patients had bowel and bladder involvement and had regained splenetic function at the final follow-up.

An inconclusive microbiological pus culture report is a dilemma for the treating surgeon. A number of reasons for this culture-negative status in biopsy-proven infection can be suggested. One possibility is that the infection has been partially treated. Kim et al. $[5,6]$ in their retrospective analysis of 53 patients with pyogenic osteomyelitis suggested that patients who received antibiotics prepresentation were most likely to have negative cultures. Our institution is a tertiary care referral hospital, with a mean delay in presentation of all patients of approximately 2 months. During this period, 20 patients (32\%) were administered antibiotics by their primary treating physician. This treatment reduces the likelihood of isolating the organism. The other factor could be the technique of retrieving the tissue sample. Because of the availability of a relatively large volume of tissue in open surgeries, there is a higher likelihood of identifying the organism. The limitation of CT-guided biopsy is that it yields a relatively small volume of tissue. The reported accuracy of CTguided spinal bone biopsy is $50 \%$ to $90 \%$ [7-9]. In our institution, the majority of patients clinically suspected to have a spinal infection will undergo CT-guided biopsy.

To our knowledge, there is no standard guideline available for treating this unique set of patients. Cultures from other foci of infection, such as blood and urine, could guide in the selection of appropriate antibiotics. Discussion with the infectious diseases department can also guide in antibiotic selection. When these options are not available, antibiotics are selected empirically. Bhagat et al. [10] compared and discussed the clinical presentation and biochemical markers and outcomes in culture-positive and culture-negative patients, but they did not discuss the usage of specific antibiotics. Seyman et al. [11] retrospectively analyzed 15 patients with culture-negative PVO treated with tigecycline. They suggested that tigecycline is the drug of choice for this group of patients. However, the cost is prohibitive. In our series, we advocated treatment with 2 weeks of intravenous antibiotics and 10 weeks of oral antibiotics from our previous experience in treating pyogenic infection [12]. During the initial 2 weeks, all patients were treated with high-end intravenous antibiotics either empirically or based on previous culture from any other focus. The initial aggressive treatment was implantation in situ, and patients receiving conservative treatment were given cloxacillin/cefazolin and one fluoroquinolone (ciprofloxacin). This was followed by 10 weeks of oral antibiotics, consisting of cloxacillin/cephalexin for grampositive coverage and ciprofloxacin for gram-negative coverage. No significant drug-related complications were 
noted.

Kim et al. [13] conducted a retrospective review of patients with culture-positive and culture-negative PVO. They demonstrated that culture-negative PVO has fewer underlying associated medical comorbidities and fewer indicators of inflammatory response. However, they did not report functional and radiological outcomes in this study population. Furthermore, Kim et al. [13] included patients who had had previous spinal procedures, and hence they studied a heterogeneous cohort. The distribution of organisms would be variable in a heterogeneous group. In our series, we included only patients with PVO of hematogenous origin.

In the workup of PVO, blood and urine cultures have been recommended as a guide for antibiotic selection. In the absence of signs of systemic sepsis, such as fever and leukocytosis, and the absence of specific signs and symptoms, the need for this investigation is questionable. Looking for primary foci of infection in the absence of signs and symptoms would increase the financial burden on the patient. In our series, 15 patients (24\%) underwent blood culture, and only six of these (40\%) grew an organism from the blood that was treated with sensitive antibiotics. Blood culture was performed in a patient who presented with systemic sepsis. Our blood culture positivity rate is comparable with that in the published literature $[8,14,15]$.

A limitation of this study was that 12 of 61 patients were lost to follow-up. Another limitation was that most patients did not undergo blood or urine culture as part of the evaluation of PVO. However, strength of the study is that it is the first series that reports the antibiotic protocol for this cohort of patients with a long follow-up. The functional and radiological outcomes have not been reported for this group so far.

\section{Conclusions}

Culture-negative, biopsy-positive $\mathrm{PVO}$ is a challenging, uncommon, and underreported condition. There are no standard antibiotic guidelines available for the treatment of this group of patients. Treatment with empirical antibiotics for 12 weeks with watchful clinical and radiological follow-up yields good resolution of the disease. Further multicenter clinical research needs to be done to obtain an algorithmic treatment plan for these patients.

\section{Conflict of Interest}

No potential conflict of interest relevant to this article was reported.

\section{References}

1. Bettini N, Girardo M, Dema E, Cervellati S. Evaluation of conservative treatment of non specific spondylodiscitis. Eur Spine J 2009;18 Suppl 1:143-50.

2. Butler JS, Shelly MJ, Timlin M, Powderly WG, O’Byrne JM. Nontuberculous pyogenic spinal infection in adults: a 12-year experience from a tertiary referral center. Spine (Phila Pa 1976) 2006;31:2695700 .

3. Kehrer M, Pedersen C, Jensen TG, Lassen AT. Increasing incidence of pyogenic spondylodiscitis: a 14-year population-based study. J Infect 2014;68:31320.

4. Ratcliffe JF. Anatomic basis for the pathogenesis and radiologic features of vertebral osteomyelitis and its differentiation from childhood discitis: a microarteriographic investigation. Acta Radiol Diagn (Stockh) 1985;26:137-43.

5. Kim CJ, Song KH, Park WB, et al. Microbiologically and clinically diagnosed vertebral osteomyelitis: impact of prior antibiotic exposure. Antimicrob Agents Chemother 2012;56:2122-4.

6. Kim CJ, Kang SJ, Yoon D, et al. Factors influencing culture positivity in pyogenic vertebral osteomyelitis patients with prior antibiotic exposure. Antimicrob Agents Chemother 2015;59:2470-3.

7. Yaffe D, Greenberg G, Leitner J, Gipstein R, Shapiro M, Bachar GN. CT-guided percutaneous biopsy of thoracic and lumbar spine: a new coaxial technique. AJNR Am J Neuroradiol 2003;24:2111-3.

8. Nolla JM, Ariza J, Gomez-Vaquero C, et al. Spontaneous pyogenic vertebral osteomyelitis in nondrug users. Semin Arthritis Rheum 2002;31:271-8.

9. Lis E, Bilsky MH, Pisinski L, et al. Percutaneous CTguided biopsy of osseous lesion of the spine in patients with known or suspected malignancy. AJNR Am J Neuroradiol 2004;25:1583-8.

10. Bhagat S, Mathieson C, Jandhyala R, Johnston R. Spondylodiscitis (disc space infection) associated with negative microbiological tests: comparison of outcome of suspected disc space infections to docu- 
mented non-tuberculous pyogenic discitis. Br J Neurosurg 2007;21:473-7.

11. Seyman D, Berk H, Sepın-Ozen N, et al. Successful use of tigecycline for treatment of culture-negative pyogenic vertebral osteomyelitis. Infect Dis (Lond) 2015;47:783-8.

12. Sundararaj GD, Babu N, Amritanand R, et al. Treatment of haematogenous pyogenic vertebral osteomyelitis by single-stage anterior debridement, grafting of the defect and posterior instrumentation. J Bone Joint Surg Br 2007;89:1201-5.
13. Kim J, Kim YS, Peck KR, et al. Outcome of culturenegative pyogenic vertebral osteomyelitis: comparison with microbiologically confirmed pyogenic vertebral osteomyelitis. Semin Arthritis Rheum 2014;44:246-52.

14. Kim CJ, Song KH, Jeon JH, et al. A comparative study of pyogenic and tuberculous spondylodiscitis. Spine (Phila Pa 1976) 2010;35:E1096-100.

15. McCarthy JJ, Dormans JP, Kozin SH, Pizzutillo PD. Musculoskeletal infections in children: basic treatment principles and recent advancements. Instr Course Lect 2005;54:515-28. 